\title{
A poroelastic model for the perfusion of the lamina cribrosa in the optic nerve head
}

\author{
Paola Causin a ${ }^{\mathrm{a}}$, Giovanna Guidoboni ${ }^{\mathrm{b}, \mathrm{c}, \mathrm{d}, *}$, Alon Harris ${ }^{\mathrm{c}}$, Daniele Prada ${ }^{\mathrm{b}}$, Riccardo Sacco ${ }^{\mathrm{e}}$, \\ Samuele Terragni ${ }^{\mathrm{f}}$
}

\author{
a Dipartimento di Matematica "F. Enriques", Università degli Studi di Milano, via Saldini 50, 20133 Milano, Italy \\ ${ }^{\mathrm{b}}$ Department of Mathematical Sciences, Indiana University Purdue University, Indianapolis, 402 N. Blackford St., LD270 Indianapolis, IN, USA \\ ${ }^{\mathrm{c}}$ Eugene and Marilyn Glick Eye Institute, Indiana University, 1160 W. Michigan St., LD270 Indianapolis, IN, USA \\ d Institut de recherche en mathématiques, interactions et applications, 7 rue René Descartes, F-67084 Strasbourg Cedex, France \\ e Dipartimento di Matematica "F. Brioschi", Politecnico di Milano, Piazza Leonardo da Vinci 32, 20133 Milano, Italy \\ ${ }_{\mathrm{f}}^{\mathrm{D}}$ Degreed in Mathematical Engineering, Politecnico di Milano, Piazza Leonardo da Vinci 32, 20133 Milano, Italy
}

\begin{abstract}
A B S T R A C T
In this work we present a mathematical model for the coupling between biomechanics and hemodynam-ics in the lamina cribrosa, a thin porous tissue at the base of the optic nerve head which is thought to be the site of injury in ocular neurodegenerative diseases such as glaucoma. In this exploratory two-dimen-sional investigation, the lamina cribrosa is modeled as a poroelastic material where blood vessels are viewed as pores in a solid elastic matrix. The model is used to investigate the influence on the distribu-tions of stress, blood volume fraction (or vascular porosity) and blood velocity within the lamina cribrosa due to the application of different levels of the intraocular pressure (IOP) and the enforcement of different mechanical constraints at the lamina's boundary. The model simulations suggest that the degree of fixity of the boundary constraint strongly influences the lamina's response to IOP elevation. Specifically, when the boundary is mechanically clamped, IOP elevation leads to an increase in stress close to the lamina's boundary, making it more susceptible to tissue damage. On the other hand, when rotations are allowed at the boundary, the most vulnerable region appears to be located at the lamina's central axis, in proximity of the eye globe, where increased stress and reduced vascular porosity and blood velocity are predicted for increased levels of IOP.
\end{abstract}

Keywords:

Biological tissues

Eye

Optic nerve head

Lamina cribrosa

Poroelastic models

Finite element method

\section{Introduction}

Elevated intraocular pressure (IOP) is one of the major risk factors for the irreversible vision loss in glaucoma, which is the second leading cause of blindness world-wide [1]. Many studies have indicated that chronical IOP elevation induces significant structural changes in a thin porous tissue at the base of the optic nerve head called lamina cribrosa [2-6]. Elevated IOP may lead to vision loss by inducing mechanical damage on the retinal gan-glion cells axons passing through the lamina (mechanical hypoth-esis) $[7,8]$ and/or by altering the blood flow within the lamina's tissue (hemodynamical hypothesis) $[9,10]$. It is reasonable to expect that the mechanical deformations of a living tissue would affect blood flow within the tissue, and therefore the mechanical

* Corresponding author at: Department of Mathematical Sciences, Indiana University Purdue University, Indianapolis, N. Blackford St., LD270 Indianapolis, IN, USA.

Available online 19 August 2014 and hemodynamical hypotheses should be addressed as one coupled problem [5,11]. In this paper, we develop a mathematical framework that allows, for the first time, to theoretically investigate the coupling between the mechanical and hemodynamical hypotheses concerning the effect of IOP elevation on the lamina's tissue.

Various mathematical models have been developed to theoretically investigate the biomechanical hypothesis, namely the biomechanical response of the lamina cribrosa to IOP elevation. For example, linear and nonlinear elastic models for thin circular plates have been used to show that thickness, radius, and mechan-ical properties of the lamina cribrosa, along with the degree of fixity offered by the sclera, are among the major factors influencing the IOP-induced deformation of the lamina $[12,13]$. Elasticity mod-els based on finite elements have been used to simulate the biomechanics of the lamina cribrosa on real geometries (see e.g. [14-16] and in the references therein) and to study the micro-architecture of the collagen fibrils within the lamina (see e.g. $[17,6]$ and the 
references therein). The biomechanical study of the ocular posterior segment is a very active area of research and, without being exhaustive, some recent contributions can be found in [18-21] and the references therein.

The main goal of this paper is to propose a theoretical framework to investigate the relationship between blood flow and mechanical deformation within the tissue of the lamina cribrosa. Mathematical models have been proposed to investigate this biomechanics-hemodynamics relationship in the tissue of the retina (see e.g. [22-24]), of the heart (see e.g. [25,26]), of the brain (see e.g. $[27,28]$ ) and of the bones (see e.g. $[29,30])$, but, to the best of our knowledge, there are no models currently available to describe the biomechanics-hemodynamics relationship within the lamina cribrosa.

In the proposed model, the lamina cribrosa is described as a poroelastic material, where blood vessels are viewed as pores in a deformable elastic matrix. The vascular porosity of the lamina cribrosa, here defined as the blood volume fraction, is assumed to change with the local state of stress and strain, which, in turn, is determined by the intertwined response of the solid and blood phases to the fluid-mechanical environment. Because of the interplay between mechanical and haemodynamical effects in lamina cribrosa microcirculation, the vascular porosity can be considered as an index of the capability of the blood to perfuse the lamina and, for this reason, it is one of the most significant quantities investigated in the present analysis.

The mechanical parameters of the solid component of the tissue are described through a nonlinear function of the local effective stress $[13,23,31]$, while the vascular permeability is represented by a quadratic function of the vascular porosity [25]. For the numerical solution of the whole coupled system, a fixed point iter-ation algorithm of staggered type between solid displacement and fluid pressure is proposed and implemented. At each step of the staggered loop, an inner fixed-point iteration is used to solve the nonlinear mechanical problem. The algorithm is endowed with a relaxation procedure for convergence acceleration, and employs the backward Euler method for time discretization and the Galerkin finite element method on a triangular grid for space discretization.

In this paper, we explore the feasibility of the proposed modeling approach on a simplified two-dimensional geometry. A comparison between model simulations and experimental measures [32] is performed to assess the capability of our simplified model to provide physiologically reasonable deformations of the lamina cribrosa for different levels of IOP. The model is then used to estimate the effect of IOP elevation on the distribution of the fluidmechanical variables in the case of different insertion conditions of the sclera, which are possibly connected to individual-specific anatomical characteristics (for example ethnicity) and, in the same individual affected by ocular disease, to the progress of the pathology itself.

\section{Methods}

In the following, we review some fundamentals of the anatomy and physiology of the lamina cribrosa, the basic assumptions underlying our modeling approach, the mathematical description of the model and the numerical strategy for its solution.

\subsection{Anatomy and physiology of the lamina cribrosa}

The lamina cribrosa is a thin, sieve-like portion of sclera at the base of the optic nerve head, formed by a multilayered network of collagen fibers that insert into the scleral canal wall (see Fig. 1(left)).
The main functions of the lamina cribrosa are (i) to act as a scaffold for the retinal ganglion cell axons which relay the visual information from the retina to the brain; (ii) to allow the central retinal artery (CRA) and the central retinal vein (CRV) to enter and leave the intraocular space; and (iii) to stabilize the pressure difference between the intraocular pressure (IOP, baseline value $12-15 \mathrm{mmHg}$ ) in the intraocular space and the retrolaminar tissue pressure (RLTp, baseline value $7-10 \mathrm{mmHg}$ ) in the optic nerve canal (see Fig. 1(right)). Blood supply to the lamina cribrosa is provided by branches of the posterior ciliary arteries (PCAs), and therefore blood enters the lamina from its outer lateral boundary, as shown in Fig. 1(left). Blood drainage occurs through the CRV, approximately located at the centre of the lamina. It is interesting to notice that, although passing through the lamina, the CRA does not contribute to the blood circulation within the lamina, as it ensures blood supply only to the inner retinal tissue [33].

\subsection{Basic assumptions}

We model the lamina cribrosa as a poroelastic medium composed of an elastic solid (comprising collagen, elastin, extracellular matrix and neural tissue) and an interconnected vascular porous space filled by blood. Blood is treated here as a Newtonian fluid as in $[25,26]$. Throughout the remainder of the article, we adopt the point of view of the classic poroelastic theory under the assumption of reversibility of the deformations and isothermal conditions (see the fundamental works of Biot [34] and the more recent reviews $[35,36])$.

We denote by $\Omega$ the spatial domain occupied by the poroelastic medium. Since the lamina cribrosa is approximately a cylindrical structure, the reasonable assumption of axially symmetric solutions leads to consider as a simpler exploratory step the rectangular domain $(0, L) \times(-d / 2, d / 2)$ corresponding to a section of the cylinder (see Fig. 2).

Having fixed a point $\mathbf{x}=\left(x_{1}, x_{2}\right)^{T}$ in $\Omega$, we indicate by $V(\mathbf{x}, t)$ an (arbitrary) representative elementary volume (REV) centered at $\mathbf{x}$ at time $t$. Then, denoting by $V_{s}(\mathbf{x}, t)$ and $V_{f}(\mathbf{x}, t)$ the volumes occupied in $V$ by the solid and the fluid, respectively, we can define the quantity

$N(\mathbf{x}, t)=\frac{V_{f}(\mathbf{x}, t)}{V(\mathbf{x}, t)}$

representing the volumetric fraction of the fluid component, or vascular porosity, and the quantity

$\frac{V_{s}(\mathbf{x}, t)}{V(\mathbf{x}, t)}=1-N(\mathbf{x}, t)$

representing the volumetric fraction of the solid component. Notice that Eq. (2) has been obtained under the assumption of fully satu-rated mixture, namely $V_{s}(\mathbf{x}, t)+V_{f}(\mathbf{x}, t)=1$.

\subsection{Balance equations and constitutive assumptions}

Denoting by $\mathbf{u}$ the solid displacement and by $p$ the fluid pressure, in the assumptions of small deformations, negligible inertial terms, absence of body forces and volumetric fluid mass sources/sinks, the poroelastic equations describing the lamina cribrosa read:

$\nabla \cdot \boldsymbol{\sigma}=\mathbf{0}$

$\boldsymbol{\sigma}=\mathbf{S}-\alpha p \mathbf{I}$

$\mathbf{S}=\mu\left(\nabla \mathbf{u}+\nabla \mathbf{u}^{T}\right)+\lambda \nabla \cdot \mathbf{u} \mathbf{I}$,

$\frac{\partial \zeta}{\partial t}=-\nabla \cdot \mathbf{v}$ 

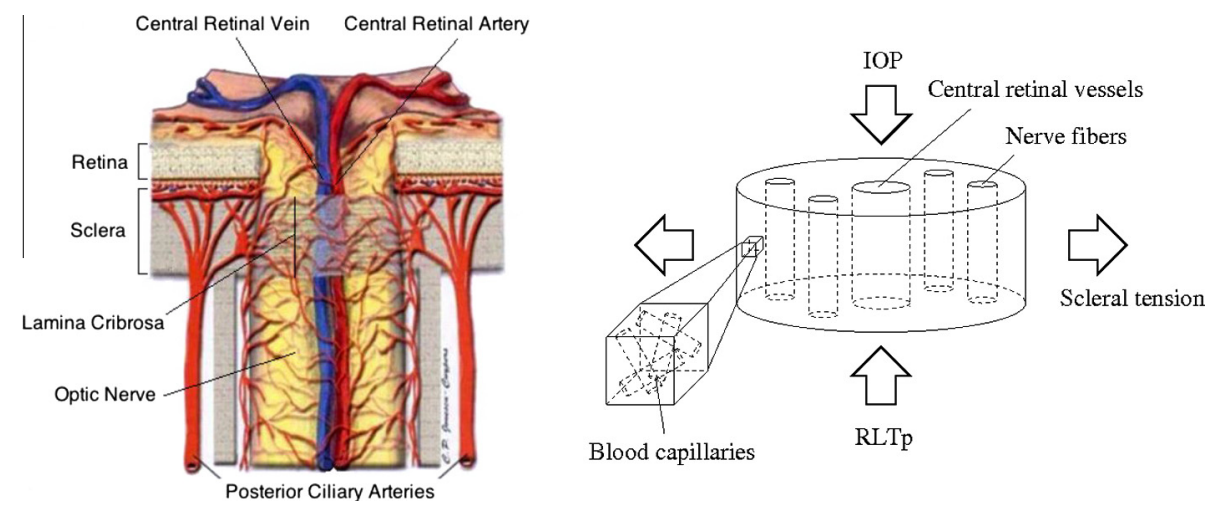

Fig. 1. Left: anatomy of the optic nerve head region with the lamina cribrosa [33]. Right: microscale view of the lamina cribrosa with the externally applied loads.

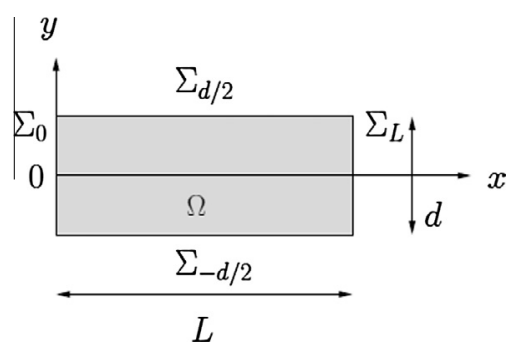

Fig. 2. Computational domain and notation for the boundaries.

$\mathbf{v}=-\mathbf{K} \nabla p$

$\zeta=\frac{1}{M} p+\alpha \nabla \cdot \mathbf{u}$

where $\boldsymbol{\sigma}$ is the stress tensor of the mixture (also known as total stress), $\mathbf{S}$ being the elastic part of the total stress, $\zeta$ is the fluid content, $\mathbf{v}$ is the discharge velocity, and $\mathbf{I}$ is the identity tensor. The discharge velocity is defined as

$\mathbf{v}(\mathbf{x}, t)=N(\mathbf{x}, t)\left(\mathbf{v}_{\text {blood }}(\mathbf{x}, t)-\mathbf{v}_{\text {solid }}(\mathbf{x}, t)\right)$,

where $\mathbf{v}_{\text {blood }}$ and $\mathbf{v}_{\text {solid }}$ are the blood and the solid velocities, respectively. The system depends on several coefficients: $\lambda$ and $\mu$, the Lamé parameters of the elastic matrix; K, the permeability tensor; $\alpha$, the Biot coefficient; and $M$, the Biot modulus.

Eqs. (3a) and (3d) express the balance of linear momentum and mass conservation, whereas Eqs. ( $3 b)$ and (3e) are the constitutive laws for the total stress and discharge velocity (Darcy's law). Eq. (3f) relates the increment in fluid content $\zeta$ to an increment in fluid pressure $p$ and a structural volumetric deformation $\nabla \cdot \mathbf{u}$, through the coefficients $M$ and $\alpha$. The solid and fluid parts of the model are coupled via the isotropic fluid stress $-\alpha p$ I in Eq. (3b), representing the contribution to the total stress due to the fluid pressure within the structure, and via the time derivative of $\zeta$ in Eq. (3d), represent-ing the variation of fluid content due to pressure and volume changes.

We assume that the solid and fluid components are incompress-ible, as it happens in the majority of biological tissues, and so we have $\alpha=1$ and $M=+\infty$ [36]. The fluid content $\zeta$ can also be expressed in terms of the vascular porosity as $\zeta=N-N_{0}$, where $N_{0}$ represents the baseline porosity value. Thus, here we assume that

$\zeta=N-N_{0}=\nabla \cdot \mathbf{u}$.

We assume that the Lamé parameter $\mu$, also known as shear modulus, varies with the effective stress $\sigma_{e}$ as originally proposed by Woo et al. [31] and adopted, for example, in [13,23], where
$\sigma_{e}=\sqrt{\frac{\left(S_{11}-S_{22}\right)^{2}+\left(S_{22}-S_{33}\right)^{2}+\left(S_{11}-S_{33}\right)^{2}+6 S_{12}^{2}}{2}}$

and $S_{i j}, i, j=1,2,3$, are the components of the stress tensor defined in Eq. (3c). The Lamé parameter $\lambda$ is derived from $\mu$ using the rela-tion $\lambda=2 \mu v /(1-2 v)$, where $v$ is the Poisson ratio, here taken equal to 0.49 . The values of $\mu$ and $\lambda$ as a function of $\sigma_{e}$ are reported in Table 1.

Remark 2.1. Other more sophisticated constitutive equations have been recently proposed to better account for the complex archi-tecture of collagen, elastin, extracellular matrix and neural fibers in the tissue of the lamina cribrosa (see e.g. $[17,6])$. These equations could be incorporated in a further extension of the model by modifying Eq. (3c).

We model the blood capillaries in the lamina cribrosa as $n_{c}$ isotropically oriented cylindrical tubes (radius $R_{c}$, length $L_{c}$ ), so that the permeability tensor can be written as $\mathbf{K}=K \mathbf{I}, K$ being a (positive) scalar function. Assuming that the capillaries change their volume at constant length, and assuming Poiseuille's flow in each capillary, it follows that the permeability varies with the square of the porosity, namely $K=\beta N^{2}$, where $\beta$ is a positive constant [25]. As estimated in a recent hystological study on the optic nerve laminar region [37], we set $N_{0}=0.156$. The constant $\beta$ is defined as the ratio between a geometric constant $c_{g}$ and the blood viscosity in the capillaries $\mu_{b}$, namely $\beta=c_{g} / \mu_{b}$. In the case of the lamina cribrosa, we choose $c_{g}=6.25 \cdot 10^{-7} \mathrm{~cm}^{2}$ and $\mu_{b}=10.01 \mathrm{cP}$ [22].

Thus, the balance equations reduce to the following coupled system in the primary variables $\mathbf{u}$ and $p$ to be satisfied in the space-time cylinder $\Omega \times(0,+\infty)$ :

$\nabla \cdot\left(\mu\left(\sigma_{e}\right)\left(\nabla \mathbf{u}+\nabla \mathbf{u}^{T}\right)+\lambda\left(\sigma_{e}\right) \nabla \cdot \mathbf{u} \mathbf{I}\right)-\nabla p=\mathbf{0}$,

$\frac{\partial \nabla \cdot \mathbf{u}}{\partial t}+\nabla \cdot(-K(\nabla \cdot \mathbf{u}) \nabla \mathbf{p})=\mathbf{0}$,

where we emphasized the fact that the model parameters are not constant, rather, $\mu=\mu\left(\sigma_{e}\right), \lambda=\lambda\left(\sigma_{e}\right)$ and $K=K(\nabla \cdot \mathbf{u})$.

Table 1

Lamé parameters for the elastic matrix of the lamina cribrosa as in Woo et al.[31].

\begin{tabular}{lccc}
\hline$\mu(\mathrm{MPa})$ & $\lambda(\mathrm{MPa})$ & \multicolumn{2}{c}{ Range of effective stress $\sigma_{e}(\mathrm{kPa})$} \\
\cline { 3 - 4 } & & From & To \\
\hline 0.12 & 5.88 & 0.0 & 8.0 \\
0.22 & 10.78 & 8.0 & 15.0 \\
0.61 & 29.89 & 15.0 & - \\
\hline
\end{tabular}




\subsection{Boundary and initial conditions}

The left boundary $\Sigma_{0}$ corresponds to the interface with the sclera, the right boundary $\Sigma_{L}$ corresponds to the central axis of the lamina cribrosa, the upper boundary $\Sigma_{d / 2}$ faces the interior of the eye globe and the lower boundary $\Sigma_{-d / 2}$ faces the optic nerve canal. Blood flow is driven by the difference between the pressure $P_{a}$ in the branches of the posterior ciliary arteries located at $\Sigma_{0}$ and the pressure $P_{v}$ in the central retinal vein located at $\Sigma_{L}$. The boundaries $\Sigma_{d / 2}$ and $\Sigma_{-d / 2}$ are assumed to be impermeable to blood flow. On $\Sigma_{d / 2}$ and $\Sigma_{-d / 2}$ we impose a traction due to the external pressures acting on the boundaries, IOP (top) and RLTp (bottom), respectively (see Fig. 1 (right) for notation and physical interpretation of applied loads). On $\Sigma_{L}$ we impose symmetry conditions for stress and displacement. At the boundary $\Sigma_{0}$ with the sclera, we consider and compare two sets of boundary conditions:

Case 1: the lamina is clamped, resulting in a homogeneous Dirichlet condition for the displacement.

Case 2: the lamina experiences a normal tension $T$ due to the inflation of the sclera, but it cannot displace vertically, resulting in a mixed condition on stress and displacement.

The boundary conditions are summarized in Table 2 . The scleral tension $T$ is computed via Laplace's law as $T=\mathrm{IOP} \cdot R_{s} / 2 h_{s}$, where $R_{s}$ and $h_{s}$ are the radius and thickness of the sclera (modeled as a sphere), respectively. Cases 1 and 2 correspond to the two extreme situations in which the scleral insertion of the lamina is at its highest and lowest degree of fixity, respectively.

Initial conditions are required only for $\nabla \cdot \mathbf{u}$, which reduces to

$N(\mathbf{x}, 0)=N_{0}$

The well posedness of a poroelastic model similar to $((7))$ was proved by Showalter in [38]. In the next section we describe how to solve numerically the system equations illustrated in Sections 2.2, 2.3, 2.4

\subsection{Numerical method}

The poroelastic model (7) contains two sources of nonlinearity. One is represented by the nonlinear coupling between solid and fluid parts because $K=K(\nabla \cdot \mathbf{u})$. The other is represented by the nonlinear constitutive equation for the solid since $\mu=\mu\left(\sigma_{e}\right)$ and $\lambda$ $=\lambda\left(\sigma_{e}\right)$. In order to address the solid-fluid coupling nonlinearity, we use a fixed point algorithm that iterates, within the same time step, between two separate subproblems for the fluid and solid part. A further inner fixed point iteration loop is then used to solve for material nonlinearity. The Backward Euler method is used for time discretization and the Galerkin finite element method for space discretization.

Following the classical approach of [39] used in incompressible linear elasticity problems, we introduce the elastic pressure

parameter $\mathcal{P}=-\lambda\left(\sigma_{e}\right) \nabla \cdot \mathbf{u}$. Denoting by $\boldsymbol{\varepsilon}(\mathbf{u})=\left(\nabla \mathbf{u}+\nabla \mathbf{u}^{T}\right) / 2$ the infinitesimal strain tensor, the balance Eqs. (7) read:

Table 2

Summary of the boundary conditions for the solid and fluid parts in the poroelastic model for the lamina cribrosa. IOP is the intraocular pressure, RLTp is the retrolaminar tissue pressure and $\mathbf{e}_{1}$ and $\mathbf{e}_{2}$ are the unit vectors of the $x_{1}$ and $x_{2}$ axes. Notice that on $\Sigma_{d / 2}$ and $\Sigma_{L}$ the outward unit normal vector $\mathbf{n}$ coincides with $+\mathbf{e}_{2}$ and $+\mathbf{e}_{1}$, respectively while on $\Sigma_{-d / 2}$ and on $\Sigma_{0}$ it coincides with $-\mathbf{e}_{2}$ and $-\mathbf{e}_{1}$, respectively. This is useful for interpreting the boundary conditions in terms of normal stress and normal velocity flux.

\begin{tabular}{lll}
\hline Boundary & Boundary conditions (solid) & Boundary conditions (fluid) \\
\hline$\Sigma_{d / 2}$ & $\boldsymbol{\sigma} \mathbf{e}_{2}=-\operatorname{IOP} \mathbf{e}_{2}$ & $\mathbf{e}_{2} \cdot \nabla p=0$ \\
$\Sigma_{L}$ & $\mathbf{u} \cdot \mathbf{e}_{1}=\mathbf{0}, \mathbf{e}_{2} \cdot \boldsymbol{\sigma} \mathbf{e}_{1}=0$ & $p=P_{v}$ \\
$\Sigma_{-d / 2}$ & $\boldsymbol{\sigma} \mathbf{e}_{2}=-\mathrm{RLT}_{p} \mathbf{e}_{2}$ & $\mathbf{e}_{2} \cdot \nabla p=0$ \\
$\Sigma_{0}$ & Case $1: \mathbf{u}=0$ & $p=P_{a}$ \\
& Case $2: \mathbf{e}_{1} \cdot \boldsymbol{\sigma} \mathbf{e}_{1}=T+P_{a}, \mathbf{u} \cdot \mathbf{e}_{2}=0$ & \\
\hline
\end{tabular}

$\nabla \cdot\left(2 \mu\left(\sigma_{e}\right) \boldsymbol{\varepsilon}(\mathbf{u})-\mathcal{P} \mathbf{I}-p \mathbf{I}\right)=\mathbf{0}$

$\frac{\mathcal{P}}{\lambda\left(\sigma_{e}\right)}+\nabla \cdot \mathbf{u}=0$

$\frac{\partial}{\partial t} \frac{\mathcal{P}}{\lambda\left(\sigma_{e}\right)}+\nabla \cdot(K(\mathcal{P}) \nabla p)=0$

To describe the computational procedure, let $\Delta t$ be the time step, $t_{n}=n \Delta t, n \geqslant 0$, and $f_{n}:=f\left(\mathbf{x}, t^{n}\right)$. The fixed point iteration algorithm that we use to advance from $t_{n}$ to $t_{n+1}$ is defined as follows:

1. let $k=0, \mathbf{u}^{(0)}=\mathbf{u}_{n}, \mathcal{P}^{(0)}=\mathcal{P}_{n}, p^{(0)}=p_{n}$ and $\sigma_{e}^{(0)}=\sigma_{e n}$;

2. set $k=k+1$;

3. find $\mathbf{u}^{(k)}$ and $\mathcal{P}^{(k)}$ such that

$$
\begin{cases}\nabla \cdot\left(2 \mu\left(\sigma_{e}^{(k)}\right) \boldsymbol{\varepsilon}\left(\mathbf{u}^{(k)}\right)-\mathcal{P}^{(k)} \mathbf{I}\right)=\nabla p^{(k-1)} & \text { in } \Omega, \\ \frac{\mathcal{P}^{(k)}}{\lambda\left(\sigma_{e}^{(k)}\right)}+\nabla \cdot \mathbf{u}^{(k)}=0 & \text { in } \Omega, \\ \left(2 \mu\left(\sigma_{e}^{(k)}\right) \boldsymbol{\varepsilon}\left(\mathbf{u}^{(k)}\right)-\mathcal{P}^{(k)} \mathbf{I}-p^{(k-1)} \mathbf{I}\right) \mathbf{e}_{2}=-\mathrm{IOP} \mathbf{e}_{2} & \text { on } \Sigma_{d / 2}, \\ \left(2 \mu\left(\sigma_{e}^{(k)}\right) \boldsymbol{\varepsilon}\left(\mathbf{u}^{(k)}\right)-\mathcal{P}^{(k)} \mathbf{I}-p^{(k-1)} \mathbf{I}\right) \mathbf{e}_{2}=-\operatorname{RLTp} \mathbf{e}_{2} & \text { on } \Sigma_{-d / 2}, \\ \mathbf{e}_{2}\left(2 \mu\left(\sigma_{e}^{(k)}\right) \boldsymbol{\varepsilon}\left(\mathbf{u}^{(k)}\right)-\mathcal{P}^{(k)} \mathbf{I}\right) \mathbf{e}_{1}=0 & \text { on } \Sigma_{L}, \\ \mathbf{u}^{(k)} \cdot \mathbf{e}_{1}=0 & \text { on } \Sigma_{L}, \\ \mathbf{u}^{(k)}=\mathbf{0}(\text { Case } 1) & \text { on } \Sigma_{0}, \\ \mathbf{e}_{1}\left(2 \mu\left(\sigma_{e}^{(k)}\right) \boldsymbol{\varepsilon}\left(\mathbf{u}^{(k)}\right)-\mathcal{P}^{(k)} \mathbf{I}-p^{(k-1)} \mathbf{I}\right) \mathbf{e}_{1}=T-P_{a} \text { (Case 2) } \text { on } \Sigma_{0}, \\ \mathbf{u}^{(k)} \cdot \mathbf{e}_{2}=\mathbf{0} \text { (Case 2) } & \text { on } \Sigma_{0},\end{cases}
$$$$
\text { 4. set } K^{(k)}=\beta\left(N_{0}-\frac{\mathcal{P}^{(k)}}{\lambda\left(\sigma_{e}^{(k)}\right)}\right)^{2}=\beta\left(N^{(k)}\right)^{2}
$$

5. find $\tilde{p}^{(k)}$ such that

$$
\begin{cases}-\nabla \cdot\left(K^{(k)} \nabla \tilde{p}^{(k)}\right)=\frac{1}{\Delta t}\left(\frac{\mathcal{p}^{(k)}-\mathcal{P}_{n}}{\lambda\left(\sigma_{e}^{(k)}\right)}\right) & \text { in } \Omega \\ \mathbf{e}_{2} \cdot \nabla \tilde{p}^{(k)}=0 & \text { on } \Sigma_{d / 2} \\ \mathbf{e}_{2} \cdot \nabla \tilde{p}^{(k)}=0 & \text { on } \Sigma_{-d / 2} \\ \tilde{p}^{(k)}=P_{v} & \text { on } \Sigma_{L} \\ \tilde{p}^{(k)}=P_{a} & \text { on } \Sigma_{0}\end{cases}
$$

6. set $p^{(k)}=\omega^{(k)} \tilde{p}^{(k)}+\left(1-\omega^{(k)}\right) p^{(k-1)}$, where $\omega^{(k)}$ is the Aitken acceleration parameter;

7. set $\mathbf{v}_{B S}^{(k)}=\mathbf{v}^{(k)} / N^{(k)}$; if

$$
\begin{array}{ll}
\frac{\left\|\mathbf{u}^{(k)}-\mathbf{u}^{(k-1)}\right\|_{L^{\infty}(\Omega)}}{\left\|\mathbf{u}^{(k-1)}\right\|_{L^{\infty}(\Omega)}}<\epsilon_{\mathbf{u}}, & \frac{\left\|\mathcal{P}^{(k)}-\mathcal{P}^{(k-1)}\right\|_{L^{\infty}(\Omega)}}{\left\|\mathcal{P}^{(k-1)}\right\|_{L^{\infty}(\Omega)}}<\epsilon_{\mathcal{P}}, \\
\frac{\left\|\mathbf{v}_{B S}^{(k)}-\mathbf{v}_{B S}^{(k-1)}\right\|_{L^{\infty}(\Omega)}}{\left\|\mathbf{v}_{B S}^{(k-1)}\right\|_{L^{\infty}(\Omega)}}<\epsilon_{\mathbf{v}}, & \frac{\left\|p^{(k)}-p^{(k-1)}\right\|_{L^{\infty}(\Omega)}}{\left\|p^{(k-1)}\right\|_{L^{\infty}(\Omega)}}<\epsilon_{p}
\end{array}
$$

are all verified for $\epsilon_{\mathbf{u}}=\epsilon_{\mathcal{P}}=\epsilon_{\mathbf{v}}=\epsilon_{p}=10^{-4}$, set $\mathbf{u}_{n+1}=\mathbf{u}^{(k)}$, $\mathcal{P}_{n+1}=\mathcal{P}^{(k)}$ and $p_{n+1}=p^{(k)}$, otherwise return to step 2 .

In the algorithm described above, the original problem is divided into two subproblems, which are solved iteratively at each time level. These subproblems correspond to the mechanical part (step 3) and the fluid part (step 5). When we solve the mechanical subproblem at step 3, we use the fluid pressure computed at the previous iteration, so that its gradient can be seen as an additional body force per unit volume of the porous medium. When we solve the fluid subproblem at step 5, we use the elastic pressure parameter computed at step 3 , so that the term with the approximation of the time derivative of $\mathcal{P}$ can be seen as an additional fluid source or sink. In other words, step 5 reduces to the solution of a linear Poisson problem with the fluid pressure as unknown which we solve using $\mathbb{P}_{2}$ finite elements, whereas step 3 entails the solution of a nonlinear elasticity problem which we solve using the $\mathbb{P}_{3}-\mathbb{P}_{2}$ finite element pair (which satisfies the discrete inf-sup condition) and the following fixed point algorithm: 


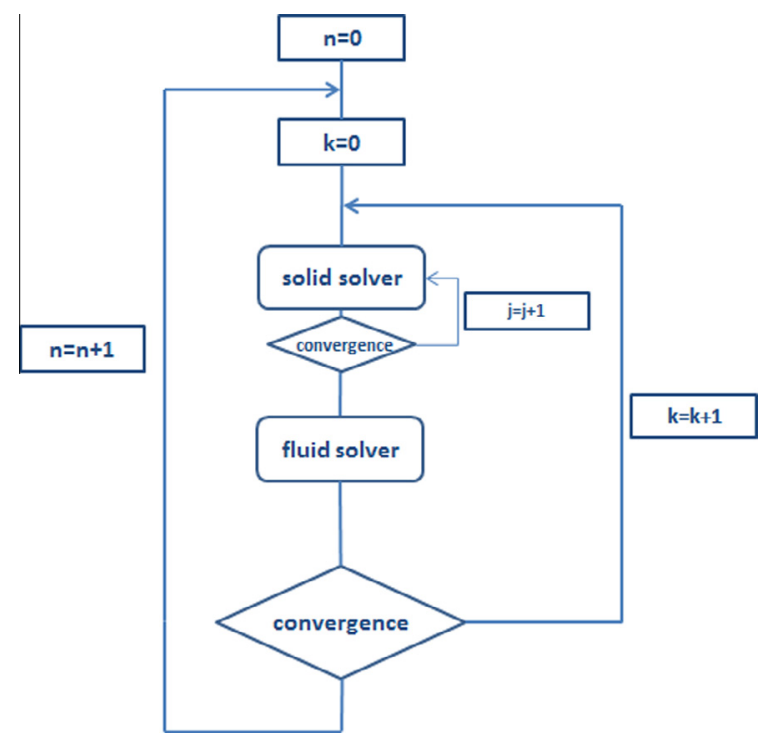

Fig. 3. A flow-chart of the computational algorithm.

1. let $j=0, \sigma_{e}^{(0)}$ be an initial guess for the effective stress, $\lambda^{(0)}=\lambda\left(\sigma_{e}^{(0)}\right), \mu^{(0)}=\mu\left(\sigma_{e}^{(0)}\right)$ and let $p^{(k-1)}$ be given;

2. set $j=j+1$;

3. find $\mathbf{u}^{(j)}$ and $\mathcal{P}^{(j)}$ such that

$$
\begin{cases}\nabla \cdot\left(2 \mu^{(j-1)} \boldsymbol{\varepsilon}\left(\mathbf{u}^{(j)}\right)-\mathcal{P}^{(j)} \mathbf{I}\right)=\nabla p^{(k-1)} & \text { in } \Omega, \\ \frac{\mathcal{P}^{(j)}}{\lambda^{(j-1)}}+\nabla \cdot \mathbf{u}^{(j)}=0 & \text { in } \Omega, \\ \left(2 \mu^{(j-1)} \boldsymbol{\varepsilon}\left(\mathbf{u}^{(j)}\right)-\mathcal{P}^{(j)} \mathbf{I}-p^{(k-1)} \mathbf{I}\right) \mathbf{e}_{2}=-\mathrm{IOP} \mathbf{e}_{2} & \text { on } \Sigma_{d / 2}, \\ \left(2 \mu^{(j-1)} \boldsymbol{\varepsilon}\left(\mathbf{u}^{(j)}\right)-\mathcal{P}^{(j)} \mathbf{I}-p^{(k-1)} \mathbf{I}\right) \mathbf{e}_{2}=-\mathrm{RLTp}_{2} & \text { on } \Sigma_{-d / 2}, \\ \mathbf{e}_{2}\left(2 \mu^{(j-1)} \boldsymbol{\varepsilon}\left(\mathbf{u}^{(j)}\right)-\mathcal{P}^{(j)} \mathbf{I}\right) \mathbf{e}_{1}=0 & \text { on } \Sigma_{L}, \\ \mathbf{u}^{(j)} \cdot \mathbf{e}_{1}=0 & \text { on } \Sigma_{L}, \\ \mathbf{u}^{(j)}=\mathbf{0}(\text { Case } 1) & \text { on } \Sigma_{0}, \\ \mathbf{e}_{1}\left(2 \mu^{(j-1)} \boldsymbol{\varepsilon}\left(\mathbf{u}^{(j)}\right)-\mathcal{P}^{(j)} \mathbf{I}-p^{(k-1)} \mathbf{I}\right) \mathbf{e}_{1}=T-P_{a} \text { (Case 2) } & \text { on } \Sigma_{0}, \\ \mathbf{u}^{(j)} \cdot \mathbf{e}_{2}=\mathbf{0} \text { (Case 2) } & \text { on } \Sigma_{0},\end{cases}
$$

4. compute $\sigma_{e}^{(j)}=\sigma_{e}\left(\mathbf{u}^{(j)}, \mathcal{P}^{(j)}\right)$

5. if $\left\|\sigma_{e}^{(j)}-\sigma_{e}^{(j-1)}\right\|_{L^{\infty}(\Omega)}<\epsilon_{\sigma_{e}}$ is verified for $\epsilon_{\sigma_{e}}=10^{-4}$, set $\mathbf{u}_{k}=\mathbf{u}^{(j)}$ and $\mathcal{P}_{k}=\mathcal{P}^{(j)}$, otherwise return to step 2 .

The time advancing procedure and the outer and inner iteration loops constituting the computational solution map for the poroelastic model (7) are schematically represented in Fig. 3.

Remark 2.2. The computational algorithm described above to advance from time level $t_{n}$ to $t_{n+1}$ shares a close structural similarity with the Gummel fixed-point iteration used in contem-porary numerical solvers for the Drift-Diffusion equation system in semiconductor device modeling [40]. This analogy may be profitably used for a theoretical analysis of existence and uniqueness of a fixed point $\left(\mathbf{u}^{*}, p^{*}\right)$ of our proposed method, and, consequently, of a solution of the nonlinear boundary value problem (9).

Remark 2.3. The choice of a staggered computational method instead of a monolithic approach to solve the coupled problem is motivated by the nonlinear elastic behavior of the solid component. The staggered scheme allows to isolate the elastic nonlinearity in a separate step and to treat it with an appropriate numerical method. This is particularly important in the view of extending the model to more realistic and complex constitutive equations for the solid component, as mentioned in Remark 2.1.

\section{Results}

In this section we compare the model simulations with experimental data [32] and then we use the model to simulate two different mechanical configurations of the lamina, corresponding to Cases 1 and 2 of Table 2, for various IOP levels. The time step used in the numerical experiments is $\Delta t=10^{-4} \mathrm{~s}$, while the horizontal and vertical dimensions of the lamina are $L=0.075 \mathrm{~cm}$ and $d=0.02 \mathrm{~cm}$, respectively. The average spatial grid size $h$ in the finite element triangulation of $\Omega$ is $3 \cdot 10^{-3} \mathrm{~cm}$. The retrolaminar tissue pressure has always been set to RLTp $=7 \mathrm{mmHg}$ [23]. Given the stationary boundary conditions we have imposed, we let our staggered method run until a stationary solution, with a constant solid displacement field, is obtained. In this case, from Eq. (4), we obtain

$\mathbf{v}(\mathbf{x})=N(\mathbf{x}) \mathbf{v}_{\text {blood }}(\mathbf{x})$.

\subsection{Comparison with experimental data}

Even though our study has to be considered preliminary with respect to the more rigorous description of the physical problem by means of cylindrical coordinates, it is still useful to make a comparison between model predictions and experimental data, in order to understand the degree of significance of our analysis.

We consider the work by Yan et al. [32], who mounted three enucleated human eyes on a specially designed experimental apparatus, which allowed to sequentially increase the IOP from 5 $\mathrm{mmHg}$ to 15,30 and $50 \mathrm{mmHg}$. Topographic images of the optic nerve head were taken at each pressure using a scanning laser tomographer (Heidelberg Retina Tomograph - HRT). These images were analysed using selected standard parameters computed by the HRT software. One of the most significant stereometric parameters is the volume below reference (VBR), defined as the volume between the cupped (with respect to the eye globe) inner surface of the lamina cribrosa and the reference plane (line $y=+d / 2$ ). VBR gives a volumetric measure of the cupping of the lamina crib-rosa and has been recommended as one of the most useful clinical measures of the optic nerve head [41].

Fig. 4 reports the comparison between the values of the IOPinduced increments of VBR, $\triangle \mathrm{VBR}$, measured experimentally for the three eyes and predicted by our mathematical model in Cases 1 and 2. More precisely, for a given IOP, the corresponding VBR increment is defined as the difference between the value of VBR for that given IOP and the value of VBR obtained for IOP equal to $5 \mathrm{mmHg}$. The values of $\triangle \mathrm{VBR}$ predicted by the model fall within the range of values measured experimentally. In particular, we notice that the slope of the segments connecting simulated data for increasing IOP values is not constant. This is a consequence of the nonlinear constitutive equation adopted for the solid compo-nent, where the Lamé parameters vary with the local effective stress. The experimental data exhibit a similar change in slope with increasing IOP, even though the data show significant variability between eyes. Despite the many simplifying assumptions embed-ded in the model, most importantly the choice of a two-dimensional geometry, the qualitative agreement between experimental data and numerical simulations is satisfactory. This suggests that our model for the lamina cribrosa provides physiologically reasonable results when comparing the response to different levels of IOP.

\subsection{Simulations of Cases 1 and 2 for various IOP values}

The poroelastic model for the lamina cribrosa is used here to estimate the effect of IOP elevation on the distributions of the fluidmechanical variables in the case of clamped boundary (Case 


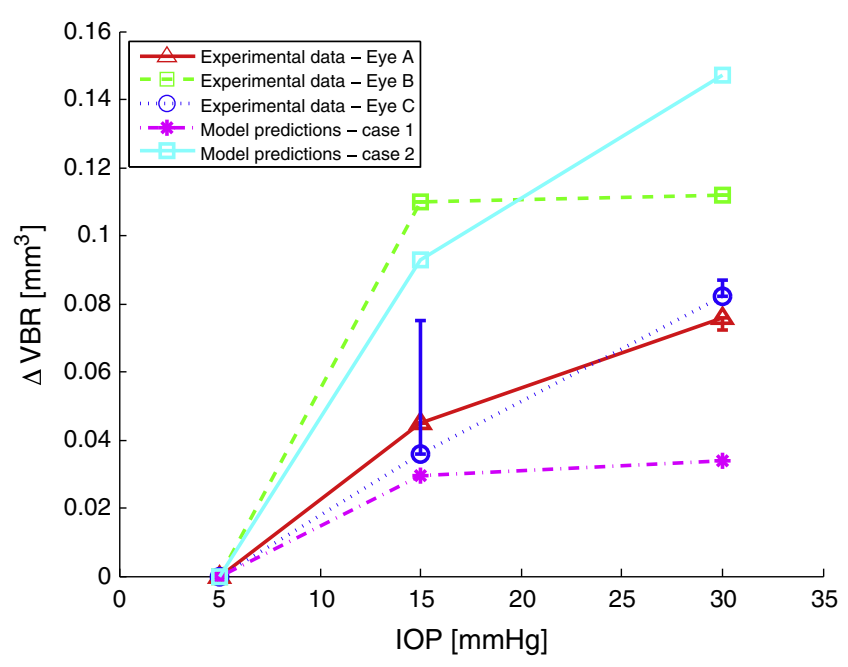

Fig. 4. Comparison between the values of the IOP-induced increments of volume below reference $(\triangle \mathrm{VBR})$ measured experimentally for the three enucleated eyes [32] and predicted by our mathematical model using the boundary conditions pertaining to Case 1 and Case 2 reported in Table 2 .

1) and in the case of imposed scleral tension and allowed rotations (Case 2).

Fig. 5 shows the deformed configuration of the lamina (red grid) in the two considered cases for IOP =15, 25 and $35 \mathrm{mmHg}$. For ease of reference, the undeformed rectangular configuration has been included as a green grid. In both conditions, computed deformations appear to be overestimated with respect to the values reported in [13], but this effect is probably to be ascribed to the combined use of a 2D geometrical model with material parameters and boundary conditions pertaining to a 3D situation. In any event, results indicate that the lamina central deflections induced by IOP elevation are larger in Case 2 than in Case 1, despite the fact that the scleral tension also increases with IOP. This difference is also reflected in the distributions of stress, porosity and blood velocity, as shown below. Blood velocity has been computed from Eq. (10). Fig. 6 compares the numerically predicted distributions of effective stress obtained in Cases 1 and 2 for IOP = 15, 25 and $35 \mathrm{mmHg}$. Results are plotted on the undeformed rectangular configuration. In the case of clamped boundary, the regions at highest $\sigma_{e}$ are located in proximity of the constraint boundary corresponding to the scleral insertion of the lamina cribrosa, namely $x_{1}$ close to 0 . When a scleral tension is imposed and rotations are allowed at the boundary, the regions at highest $\sigma_{e}$ are located in proximity of the lamina central axis, namely $x_{1}$ close to $L=0.075$, and extend further into the domain as IOP increases.

The numerically predicted distribution of the vascular porosity reported in Fig. 7 shows a similar behavior as the effective stress. IOP elevations seem to affect the lamina porosity in Case 2 more than in Case 1. In the former case, wide regions of decreased and increased porosity are apparent along the upper and lower boundaries of the lamina around its central axis.

Fig. 8 shows remarkable differences between Cases 1 and 2 also in the distribution of blood velocity. In Case 1, IOP elevation does not cause substantial variations in the velocity distribution. In Case 2 , the region of maximum velocity is always located at the lower surface of the lamina, and IOP elevation induces a velocity reduction in the top central area of the lamina.

\section{Discussion}

The main goal of this work is to develop a poroelastic model to describe the relationship between mechanical deformations and blood flow in the lamina cribrosa for increasing values of IOP, since

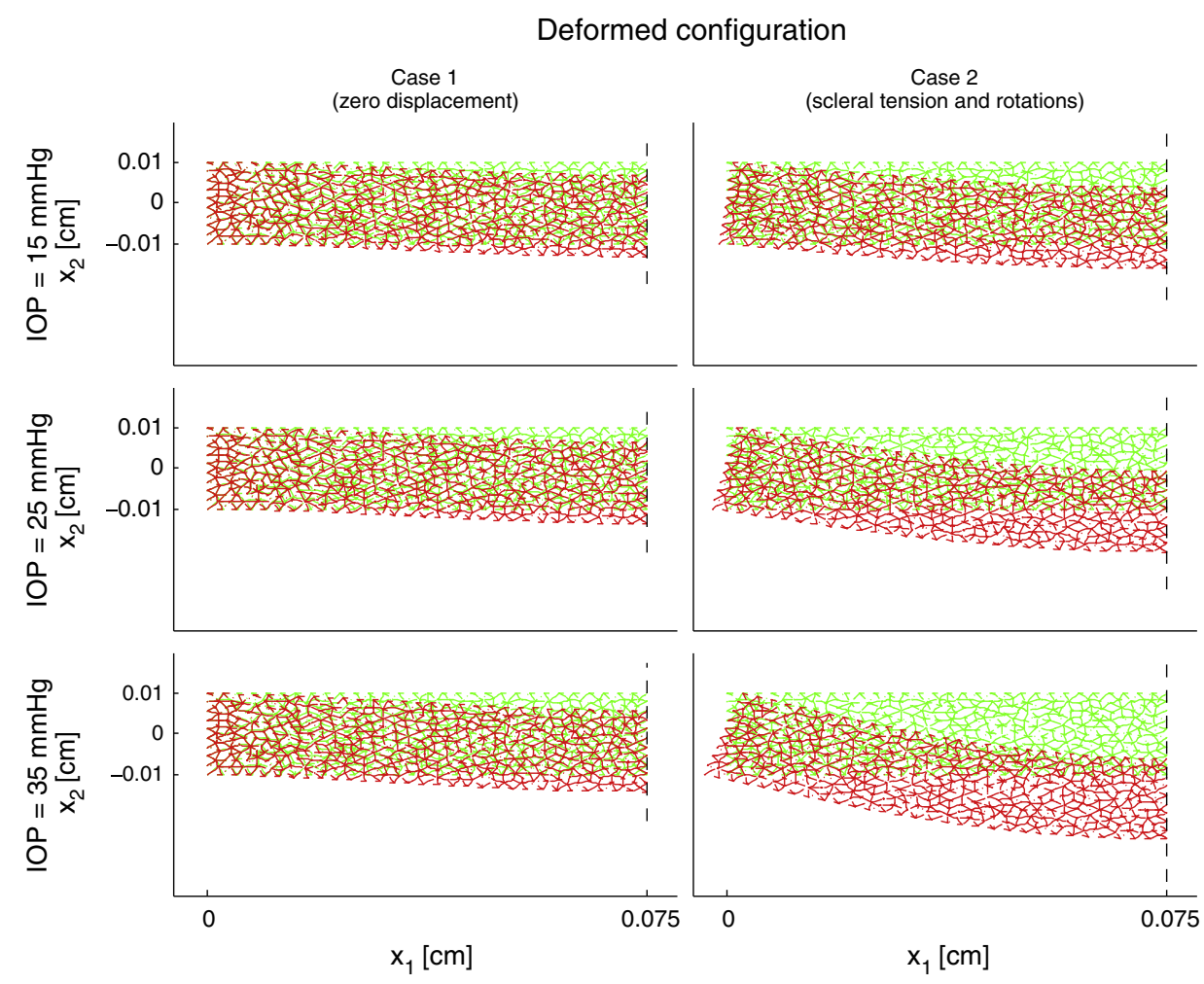

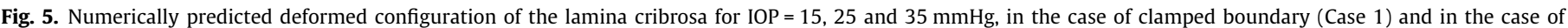

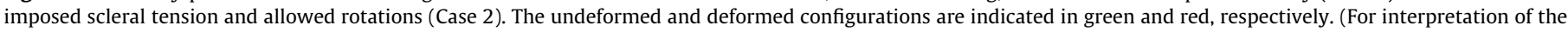
references to color in this figure legend, the reader is referred to the web version of this article.) 


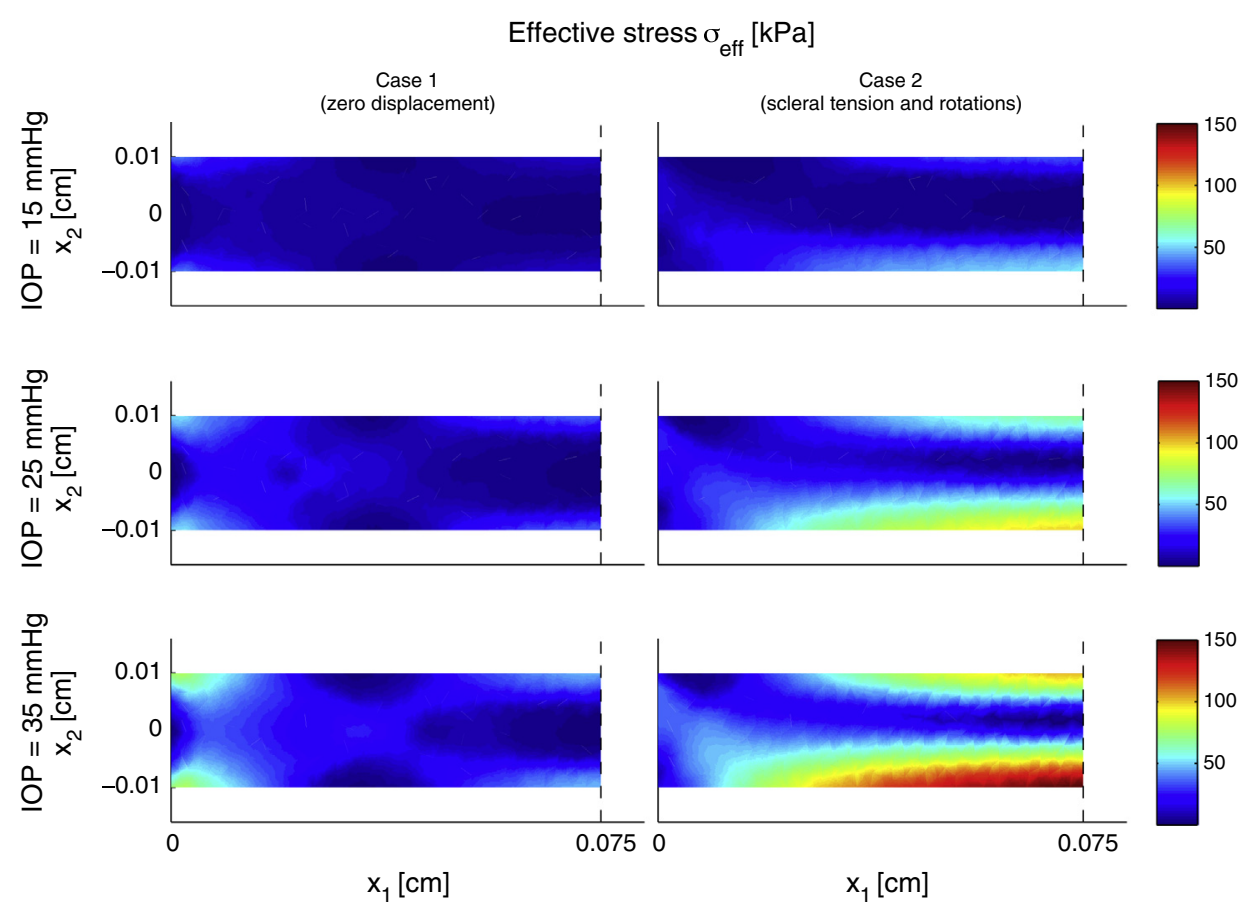

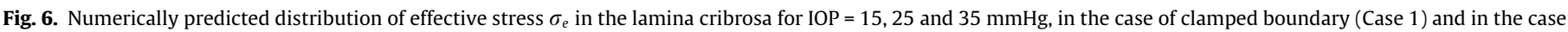
of imposed scleral tension and allowed rotations (Case 2). Results are plotted on the undeformed rectangular configuration.
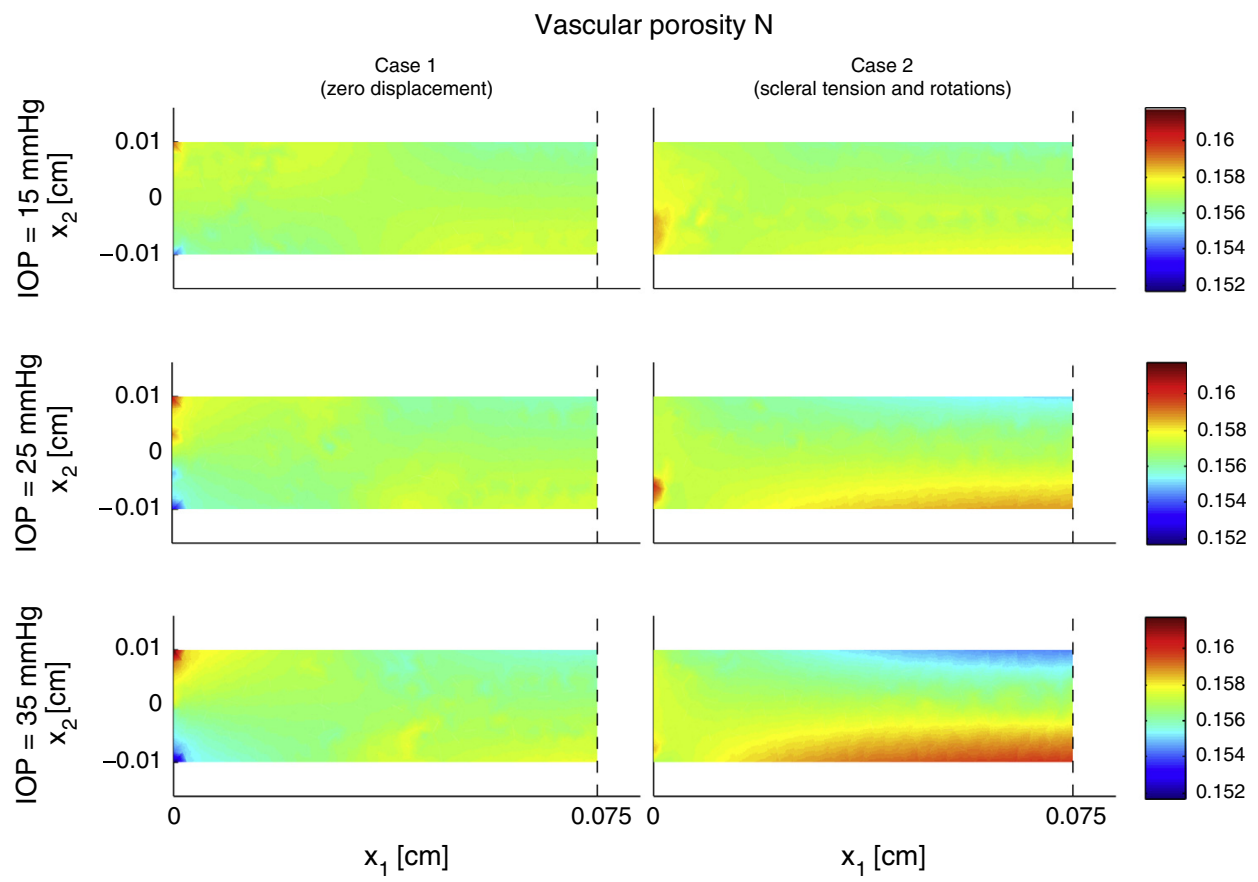

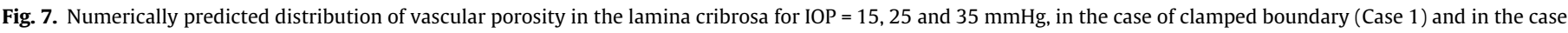
of imposed scleral tension and allowed rotations (Case 2). Results are plotted on the undeformed rectangular configuration.

this relationship is thought to play a crucial role in onset and progression of glaucoma. The model predicts that the same IOP elevation, for example from 15 to $35 \mathrm{mmHg}$, may have remarkably different biomechanical and hemodynamical consequences in the lamina cribrosa of individuals experiencing different degrees of fixity at the boundary between sclera and lamina.

In the extreme situation of clamped boundary (Case 1), the region close to the sclera is subject to higher stress and lower blood velocity than the rest of the lamina, making it more susceptible to tissue damage. On the other hand, when rotations are allowed and a scleral tension is imposed at the boundary between lamina and sclera (Case 2), the most vulnerable region is located at the upper surface close to the central axis of the lamina, whereas the lower surface experiences increased blood velocity. These findings suggest that the relationship between the mechanical deformations and the blood flow in the lamina cribrosa is far from trivial and that 


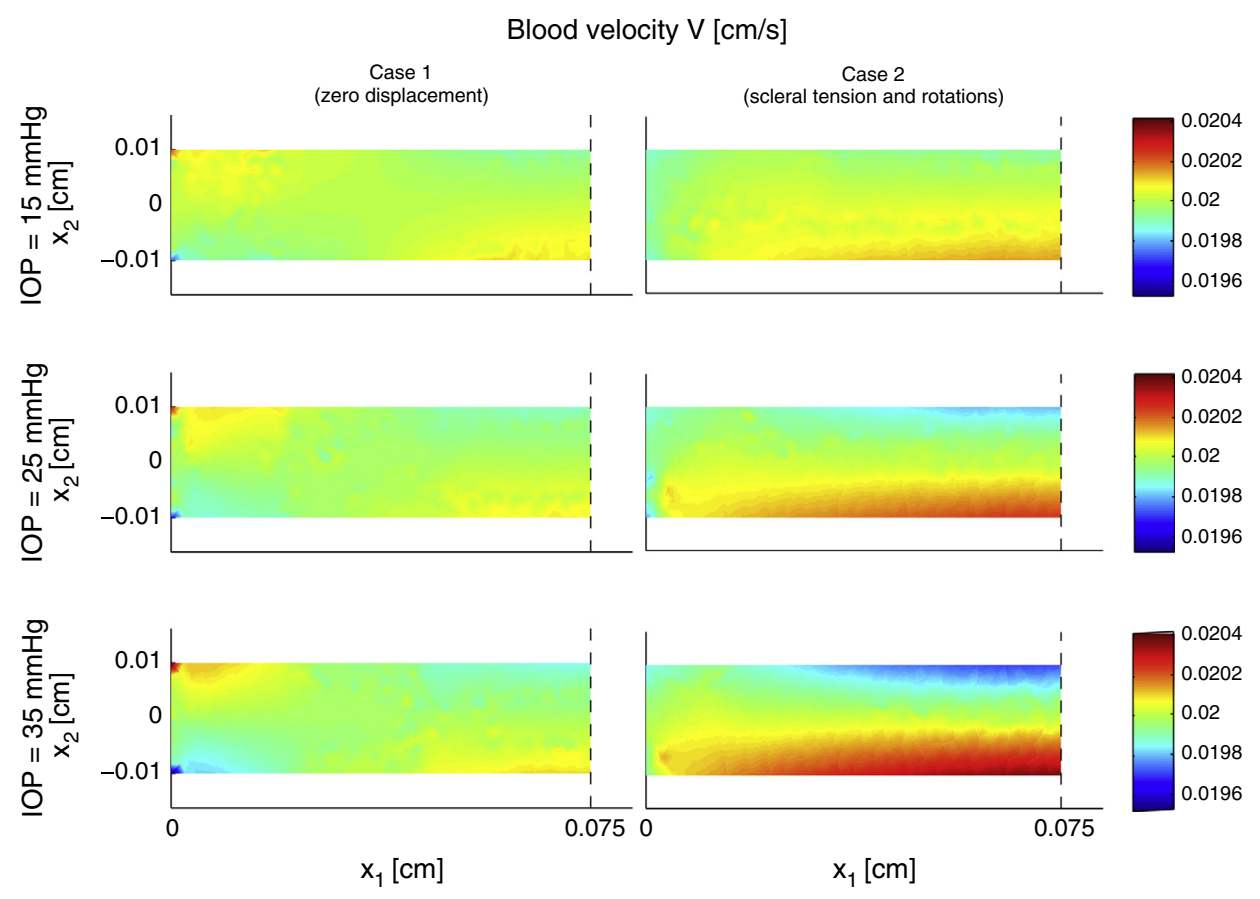

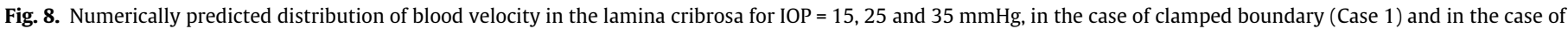
imposed scleral tension and allowed rotations (Case 2). Results are plotted on the undeformed rectangular configuration.

the use of mathematical modeling could help identifying the main factors that influence such relationship.

It is important to remind once again that the model simulations have been obtained under many simplifying assumptions, most importantly we have considered a simplified two-dimensional geometry and a trilinear constitutive equation for the laminar tissue. Despite the many limitations of the model, it is interesting to notice how the model predicts different biomechanical and hemodynamic responses to IOP elevation depending on the region within the lamina and the degree of fixity of the scleral insertion. Many clinical and experimental observations suggest that the response of the laminar tissue to changes in IOP is not uniform. For example, loss of collagen fibers was reported in the top-centre of the lamina and recruitment of new collagen was detected at the lower surface, causing cupping in the lamina of animals suffering from prolonged IOP elevation [5]. It would be interesting to further develop the model to investigate whether and to what extent this non-uniform growth and remodeling of the collagen within the lamina is mediated by blood perfusion and availability of nutrients.

Many factors influence the properties of the scleral insertion of the lamina cribrosa, such as ethnicity [42] and age 43]. In addition, these properties have observed to change within the same individual as glaucoma progresses [44]. It would be interesting to further extend the model to investigate other types of boundary conditions at the scleral insertion, such as, for example, compliant conditions by means of combinations of springs and supports. Moreover, IOP and arterial blood pressure $P_{a}$ are known to vary over a cardiac cycle as well as during the day. The influence of time variations in IOP and $P_{a}$ on the blood flow through the lamina would be another very interesting direction of research that could be investigated using our model.

\section{Acknowledgements}

This work was partially supported by the NSF/DMS Grants 1134731 and 1224195, the NIH Grant 1R21EY022101-01A1.

\section{References}

[1] M.C. Leske, Open-angle glaucoma: an epidemiologic overview, Ophthalmic. Epidemiol. 14 (2007) 166-172.

[2] A. Bhole, B. Flynn, M. Liles, N. Saeidiand, C. Dimarzio, J. Ruberti, Mechanical strain enhances survivability of collagen micronetworks in the presence of collagenase: implications for load-bearing matrix growth and stability, Philos. Trans. Roy. Soc. A 367 (2009) 3339-3362.

[3] R. Camp, M. Liles, J. Beale, N.S.B. Flynn, E. Moore, S. Murthy, J. Ruberti, Molecular mechanochemistry: low force switch slows enzymatic cleavage of human type I collagen monomer, J. Am. Chem. Soc. 133 (2011) 4073-4078.

[4] B. Flynn, A.B.N. Saeidi, M. Liles, C. Dimarzio, J. Ruberti, Mechanical strain stabilizes reconstituted collagen fibrils against enzymatic degradation by mammalian collagenase matrix metalloproteinase 8 ( $\mathrm{mmp}-8$ ), PLoS ONE 5 (2010) e12337.

[5] R. Grytz, C. Girkin, V. Libertiaux, J. Downs, Perspectives on biomechanical growth and remodeling mechanisms in glaucoma, Mech. Res. Commun. 42 (2012) 92-106.

[6] R. Grytz, M. Fazio, M. Girard, V. Libertiaux, L. Bruno, S. Gardiner, C. Girkin, J Downs, Material properties of the posterior human sclera, J. Mech. Behav. Biomed. Mater. 29 (2014) 602-617.

[7] C. Burgoyne, J. Downs, A. Bellezza, J.-K.F. Suh, R.T. Hart, The optic nerve head as a biomechanical structure: a new paradigm for understanding the role of IOP related stress and strain in the pathophysiology of glaucomatous optic nerve head damage, Prog. Retin. Eye Res. 24 (1) (2005) 39-73.

[8] C. Burgoyne, A biomechanical paradigm for axonal insult within the optic nerve head in aging and glaucoma, Exp. Eye Res. 93 (2) (2011) $120-132$.

[9] J. Caprioli, A.L. Coleman, Blood pressure, perfusion pressure, and glaucoma, Am. J. Ophthalmol. 149 (5) (2010) 704-712.

[10] R. Weinreb, A. Harris (Eds.), Ocular Blood Flow in Glaucoma, Kugler Publications, 2009.

[11] A. Harris, G. Guidoboni, J.C. Arciero, A. Amireskandari, L.A. Tobe, B.A. Siesky, Ocular hemodynamics and glaucoma: the role of mathematical modeling, Eur. J. Ophthalmol. 23 (2) (2013) 139-146.

[12] H. Dongqi, R. Zeqin, A biomathematical model for pressure-dependent lamina cribrosa behavior, J. Biomech. 32 (6) (1999) 579-584.

[13] T. Newson, A. El-Sheikh, Mathematical modeling of the biomechanics of the lamina cribrosa under elevated intraocular pressures, J. Biomech. Eng. 128 (4) (2006) 496-504.

[14] R. Norman, J. Flanagan, I. Sigal, S. Rausch, I. Tertinegg, C.R. Ethier, Finite element modeling of the human sclera: influence on optic nerve head biomechanics and connections with glaucoma, Exp. Eye Res. 93 (1) (2011) 4-12.

[15] I. Sigal, J. Flanagan, C. Ethier, Factors influencing optic nerve head biomechanics, Invest. Ophthalmol. Vis. Sci. 46 (11) (2005) 4189-4199. 
[16] I. Sigal, H. Yang, M. Roberts, C. Burgoyne, J. Downs, IOP-induced lamina cribrosa displacement and scleral canal expansion: an analysis of factor interactions using parameterized eye-specific models, Invest. Ophthalmol. Vis. Sci. 52 (3) (2011) 1896-1907.

[17] R. Grytz, G. Meschke, J.B. Jonas, The collagen fibril architecture in the lamina cribrosa and peripapillary sclera predicted by a computational remodeling approach, Biomech. Model. Mechanobiol. 10 (3) (2011) 371-382.

[18] I. Campbell, B. Coudrillier, C. Ethier, Biomechanics of the posterior eye: a critical role in health and disease, J. Biomech. Eng. 136 (2) (2014) 021005.

[19] H. Morris, J. Tang, B.C. Perez, X. Pan, R. Hart, P. Weber, J. Liu, Correlation between biomechanical responses of posterior sclera and IOP elevations during micro intraocular volume change, Invest. Ophthalmol. Vis. Sci. 54 (12)(2013) 7215-7222.

[20] M. Girard, W. Dupps, M. Baskaran, G. Scarcelli, S. Yun, H. Quigley, I. Sigal, N. Strouthidis, Translating ocular biomechanics into clinical practice: current state and future prospects, Curr. Eye Res. 15 (2014) 1-18.

[21] I. Sigal, J. Grimm, J. Schuman, L. Kagemann, H. Ishikawa, G. Wollstein, A method to estimate biomechanics and mechanical properties of optic nerve head tissues from parameters measurable using optical coherence tomography, IEEE Trans. Med. Imag. 33 (6) (2014) 1381-1389.

[22] J. Arciero, A. Harris, B. Siesky, A. Amireskandari, V. Gershuny, A. Pickrell, G. Guidoboni, Theoretical analysis of vascular regulatory mechanisms contributing to retinal blood flow autoregulation, Invest. Ophthalmol. Vis. Sci. 54 (8) (2013) 5584-5593.

[23] G. Guidoboni, A. Harris, L. Carichino, Y. Arieli, B.A. Siesky, Effect of intraocular pressure on the hemodynamics of the central retinal artery: a mathematical model, Math. Biosci. Eng. 11 (3) (2014) 523-546.

[24] G. Guidoboni, A. Harris, S. Cassani, J. Arciero, B. Siesky, A. Amireskandari, L. Tobe, P. Egan, I. Januleviciene, J. Park, Intraocular pressure, blood pressure and retinal blood flow autoregulation: a mathematical model to clarify their relationship and clinical relevance, Invest. Ophthalmol. Vis. Sci. 55 (7) (2014) 4105-4118.

[25] J. Huyghe, T. Arts, D. van Campen, R. Reneman, Porous medium finite element model of the beating left ventricle, Am. J. Physiol. 262 (4 Pt 2) (1992) H1256H1267.

[26] D. Chapelle, J. Sainte-Marie, J.-F. Gerbeau, I. Vignon-Clementel, A poroelastic model valid in large strains with applications to perfusion in cardiac modeling, Comput. Mech. 46 (1) (2010) 91-101.

[27] B. Tully, Y. Ventikos, Cerebral water transport using multiplenetwork poroelastic theory: application to normal pressure hydrocephalus, J. Fluid Mech. 667 (2011) 188-215.
[28] X. Li, H. von Holst, S. Kleiven, Influences of brain tissue poroelastic constants on intracranial pressure (icp) during constant-rate infusion, Comput. Methods Biomech. Biomed. Engin. 16 (12)(2013) 1330-1343.

[29] S. Cowin, Bone poroelasticity, J. Biomech. 32 (3) (1999) 217-238.

[30] Y. Yoon, J. Chung, C. Bae, S. Han, The speed of sound through trabecular bone predicted by Biot theory, J. Biomech. 45 (4) (2012) 716-718.

[31] S.L.-Y. Woo, A.S. Kobayashi, W.A. Schlegel, C. Lawrence, Nonlinear material properties of intact cornea and sclera, Exp. Eye Res. 14 (1) (1972) 29-39.

[32] D.B. Yan, J.G. Flanagan, T. Farra, G.E. Trope, C.R. Ethier, Study of regional deformation of the optic nerve head using scanning laser tomography, Curr. Eye Res. 17 (9) (1998) 903-916.

[33] A. Harris, C. Jonescu-Cuypers, L. Kagemann, T. Ciulla, G. Krieglstein, Atlas of Ocular Blood Flow, Vascular Anatomy, Pathophysiology, and Metabolism, Elsevier, 2003.

[34] M.A. Biot, General theory of three-dimensional consolidation, J. Appl. Phys. 12 (2) (1941) 155-164

[35] O. Coussy, Poromechanics, John Wiley \& Sons, 2004.

[36] E. Detournay, A.H.-D. Cheng, Fundamentals of poroelasticity, in: C. Fairhurst (Ed.), Comprehensive Rock Engineering: Principles, Practice and Projects, vol. II, Pergamon Press, 1993, pp. 113-171 (Chapter 5).

[37] C. Balaratnasingam, M. Kang, P. Yu, G. Chan, W. Morgan, S. Cringle, D. $\mathrm{Yu}$, Comparative quantitative study of astrocytes and capillary distribution in optic nerve laminar regions, Exp. Eye Res. 121 (2014) 11-22.

[38] R.E. Showalter, Diffusion in poro-elastic media, J. Math. Anal. Appl. 251 (1)(2000) 310-340.

[39] L.R. Herrmann, Elasticity equations for incompressible and nearly incompressible materials by a variational theorem, AIAA J. 3 (10) (1965) 1896-1900

[40] J. Jerome, Analysis of Charge Transport: A Mathematical Study of Semiconductor Devices, Springer-Verlag, 1996.

[41] W. Hatch, J. Flanagan, E. Etchells, D. Williams-Lyn, G. Trope, Laser scanning tomography of the optic nerve head in ocular hypertension and glaucoma, Br. J. Ophthalmol. 81 (10) (1997) 871-876.

[42] G. Guidoboni, A. Harris, J. Arciero, B. Siesky, A. Amireskandari, A. Gerber A. Huck, N. Kim, S. Cassani, L. Carichino, Mathematical modeling approaches in the study of glaucoma disparities among people of african and european descents, J. Coupled Syst. Multiscale Dyn. 1 (1) (2013) 1-21.

[43] M. Girard, J. Suh, M. Bottlang, C. Burgoyne, J. Downs, Scleral biomechanics in the aging monkey eye, Invest. Ophthalmol. Vis. Sci. 50 (11)(2009) 52265237.

[44] J. Jonas, L. Holbach, S. Panda-Jonas, Peripapillary ring: histology and correlations, Acta Ophthalmol. 92 (4) (2014) e273-e279. 